\title{
Measurement of the mapping between intracranial EEG and fMRI recordings in the human brain
}

Carmichael DW ${ }^{1,2}$, Vulliemoz $\mathrm{S}^{2,3}$, Murta $\mathrm{T}^{2,4}$, Chaudhary U. ${ }^{2}$, Perani $\mathrm{S}^{1}$,

Rodionov $\mathrm{R}^{2}$, Rosa $\mathrm{MJ}^{5}$, Friston $\mathrm{KJ}^{6}$, Lemieux $\mathrm{L}^{2}$

1. Developmental Imaging and Biophysics section, UCL Great Ormond Street Institute of Child Health, London, WC1N 1EH.

2. Department of Clinical and Experimental Epilepsy, UCL Institute of Neurology, Queen Square, London, WC1E 6BT

3. Epilepsy Unit, Neurology Department, University Hospital and University of Geneva, 1211 Geneva 14, Switzerland.

4. Department of Bioengineering, Institute for systems and robotics, Instituto Superior Tecnico, Universidade de Lisboa, Lisbon, Portugal, 1049-001

5. Department of Computer Science, UCL, London, UK

6. Wellcome Trust Centre for Neuroimaging, UCL Institute of Neurology, Queen Square, London

Corresponding author: David Carmichael

Correspondence to:

UCL Institute of Child Health

30 Guilford Street

London WC1N 1EH

Tel +44 (0)2079052298

Fax +44 (0)20 79052358

email d.carmichael@ucl.ac.uk 


\section{Abstract (150 non-technical)}

There are considerable gaps in our understanding of the relationship between human brain activity measured at different temporal and spatial scales by intracranial electroencephalography and $\mathrm{fMRI}$. By comparing individual features and summary descriptions of intracranial EEG activity we determined which best predict fMRI changes in the sensorimotor cortex in two brain states: at rest and during motor performance. We also then examine the specificity of this relationship to spatial colocalisation of the two signals.

We acquired electrocorticography and fMRI simultaneously (ECoG-fMRI) in the sensorimotor cortex of 3 patients with epilepsy. During motor activity, high gamma power was the only frequency band where the electrophysiological response was colocalised with fMRI measures across all subjects. The best model of fMRI changes was its principal components, a parsimonious description of the entire ECoG spectrogram. This model performed much better than a model based on the classical frequency bands both during task and rest periods or models derived on a summary of cross spectral changes (e.g. 'root mean squared EEG frequency' ). This suggests that the region specific fMRI signal is reflected in spatially and spectrally distributed EEG activity. 


\section{INTRODUCTION}

Functional Magnetic Resonance Imaging (fMRI) has emerged as the pre-eminent neuroimaging modality for studying functional segregation - and increasingly integration (1) - reflecting its capacity to map distributed hemodynamic (blood oxygen level dependent or 'BOLD' signal) changes at the scale of millimetres over the entire human brain. Although BOLD signal changes are considered a marker of underlying neuronal activity, the neuronal basis of the fMRI signal remains the subject of intense investigation. This is, largely because of its importance for understanding, modelling and inferring underlying brain processes (2, 3). Animal studies have shown that BOLD signal changes are most closely related to local field potentials in the high gamma-band range (4) and reflect input and intra-cortical processing (3). However, there are discrepancies regarding which frequency band (3-5) or combination of frequency bands (6-8) is the best correlate of the BOLD signal, which could be task, species or brain system dependent (9). There is further uncertainty about how BOLD responses relate to electrophysiological activity occurring at different frequencies, with both BOLD decreases and increases (relative to a task or state baseline) reported $(10,11)$. Previous studies have largely focused on particular frequencies or classical EEG frequency bands to predict BOLD changes, however, state-related EEG changes are often cross-spectral $(12,13)$. There have been reports of BOLD changes being well represented by cross-spectral metrics of overall EEG signal change such as the 'root mean square frequency' (13), however, these models have not been tested in data with a wide spectral range or in different states such as during rest.

Furthermore, the spatial aspect of this relationship is relatively neglected. For example, fluctuations in oscillatory activity, which may be related to information transfer and network control $(14,15)$, recorded in one location, could index synaptic and metabolic changes in a remote region. Additionally, the spatial extent of electrophysiological activity (e.g. motor activity and motor inhibition) is likely to be distributed over several cortical areas $(10,16)$.

Intracranial EEG recordings are performed for localisation purpose in some medically refractory patients with focal epilepsy who are candidates for epilepsy surgery; intracerebral depth electrodes that penetrate the brain (so-called 'depth EEG' or 'stereo-EEG': SEEG), provide exquisitely localised data, while subdural grid electrodes placed on the cortex (ECoG) capture activity over larger cortical regions. Importantly, these recordings do not attenuate high-frequency activity, unlike scalp EEG which suffers from inherent spatial-temporal filtering and muscle artefacts. While spatial sampling is limited by clinical considerations, icEEG has greater 
sensitivity and spatial specificity in relation to electrophysiological activity, compared to its scalp-based counterparts $(17,18)$. Finally, there is also more limited evidence of coupling between BOLD and electrophysiology in the resting state (19-21).

In this study, we used simultaneous ECoG-fMRI acquired in three patients with epilepsy during invasive pre-surgical investigations. ECoG coverage of the sensorimotor cortex provided a unique opportunity to explore the coupling between electrophysiology and $\mathrm{fMRI}$, with exquisite spatial precision and greater spectral range than available from scalp recordings, while accounting for spontaneous activity and variability both during task and rest conditions. Using these data, we were able to characterise the relationship between EEG activity and fMRI signal changes within the sensorimotor cortex during a motor task and contrast it with the equivalent coupling in the resting state. We aimed to determine: 1) the best model of BOLD changes in particular comparing frequency specific and cross-spectral EEG models of $\mathrm{FMRI}$; 2) if the best model was state dependant and 3) characterise the sensitivity of this relationship to the colocalisation between the EEG and fMRI signals.

\section{RESULTS}

Frequency-specific ECoG-fMRI correlation during a motor task vs. resting state We sought to characterise the coupling between the fMRI signal in the hand sensorimotor region and spatially distributed ECoG activity as a function of frequency for two brain states: rest and motor task. This was achieved by calculating the correlation over time between the fMRI signal averaged over the task-activated hand sensorimotor region and the fluctuations in ECoG power as a function of frequency and ECoG electrode contact location, after convolution with a hemodynamic response function (HRF) (22) (fig. 1):

\section{Left $v$ right finger tap (30s blocks) $p<.05$ FWE corrected}

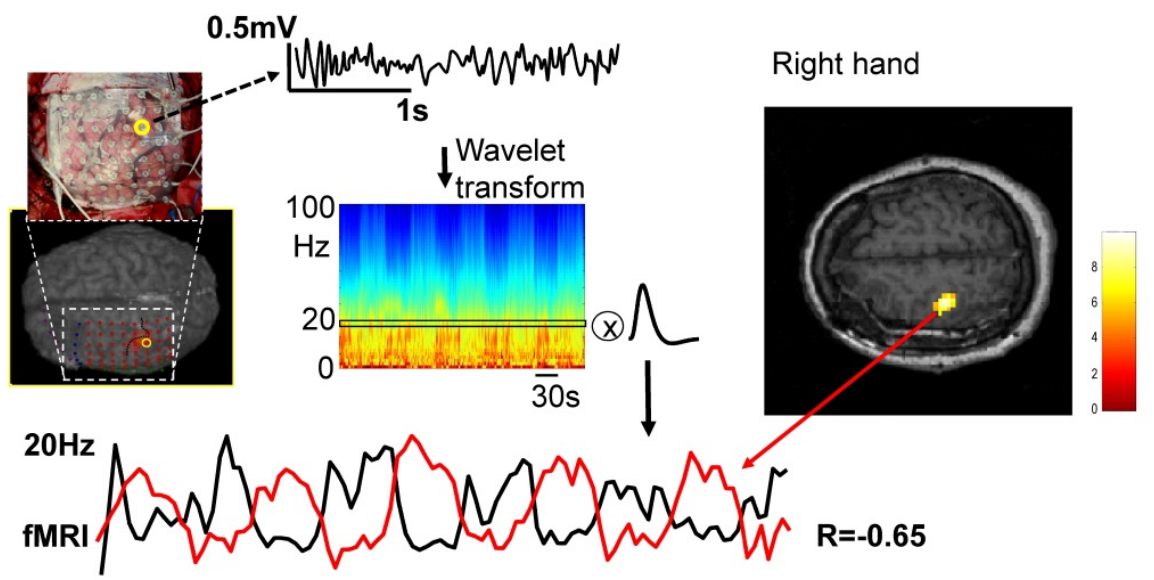

Figure 1. Analysis overview 
The process of comparing $\mathrm{fMRI}$ to ECoG data is shown with ECoG data from the post-central ECoG (contact \#22, subject \#1) transformed into time-frequency space before being convolved with the HRF. This yields a spectrally specific model of the fMRI changes (e.g. black line at the bottom) for correlation (or model comparison) with the $f M R I$ signal in the hand sensorimotor region (red line at bottom; average from task activated region, $p<0.05$ FWE corrected). This was the $\mathrm{fMRI}$ region used throughout due to the strong evidence that it is commonly active both during rest and task (23). The correlation between ECoG power at $19 \mathrm{~Hz}$ and $\mathrm{fMRI}$ signal during the task is shown to be strong and negative. This correlation analysis was repeated for each frequency during the task and rest to establish spectral specificity (see fig 2a-c) and then for each electrode contact to establish spatial specificity (see fig. 3 and 4).

First, we identified the electrode exhibiting the strongest correlation for each subject. The strongest correlation was always found at an electrode located either directly above hand primary motor cortex or immediately posterior to the central sulcus (fig. $2 a-c)$. Note that due to the spatial relationship between the ECoG grid at the surface and the cortical folding, broadly, this contact was always overlying motor cortex. During the motor task, the maximum positive correlation as a function of frequency was found in the high gamma band for all three subjects $(91 \mathrm{~Hz}$ for subject \#1 and $69 \mathrm{~Hz}$ for subjects \#2 and \#3, fig. 2a-c respectively, black) and the strongest negative correlation was found in the beta band $(17 \mathrm{~Hz}$ for subject \#1, $15 \mathrm{~Hz}$ for subject \#2 and $29 \mathrm{~Hz}$ for subject \#3). In the resting state, the profile of the coupling - as a function of frequency - was similar but the correlation was weaker and more variable between subjects (fig. $2 \mathrm{a}-\mathrm{c}$, red). The most distinctive feature of the coupling profile during rest was a significant negative correlation in the low beta range $(<20 \mathrm{~Hz})$. 
Activated region average timecourse (detrended) correlated with EEG power timecourse at $1,3,5 \ldots \ldots 99 \mathrm{~Hz}$ frequency
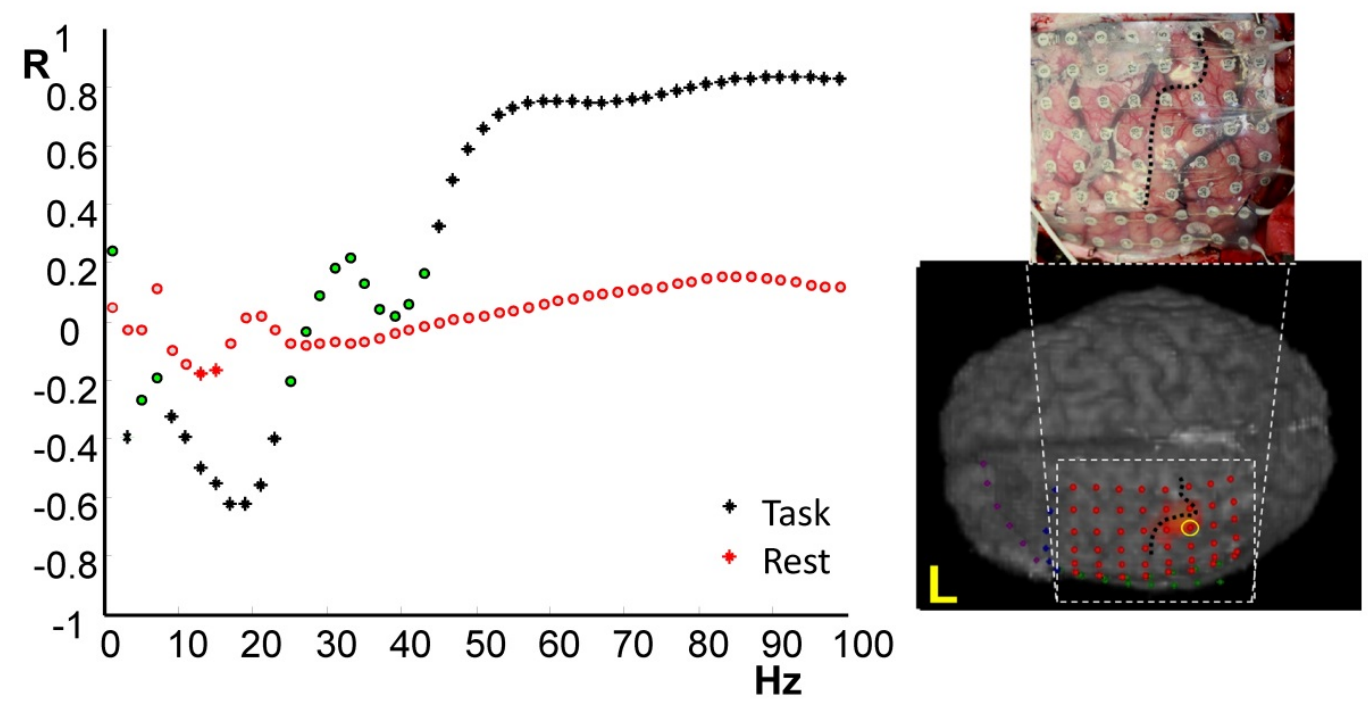

a.

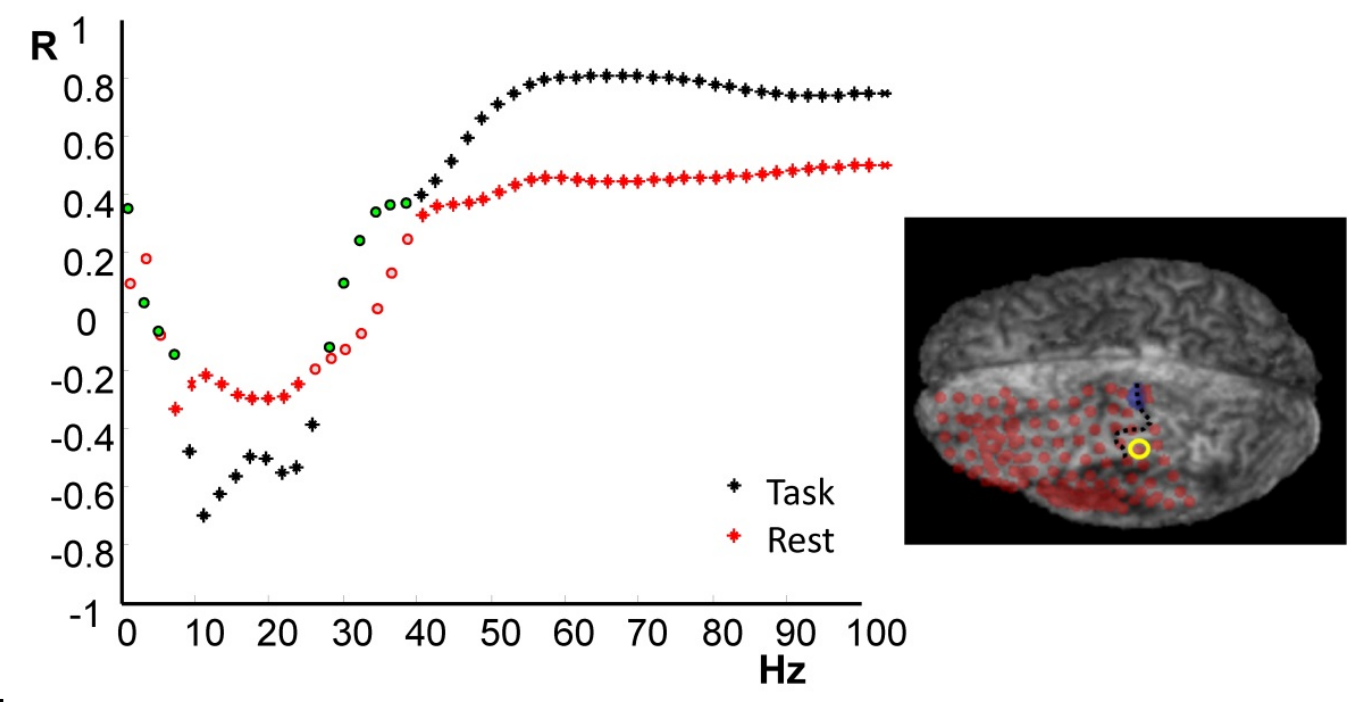



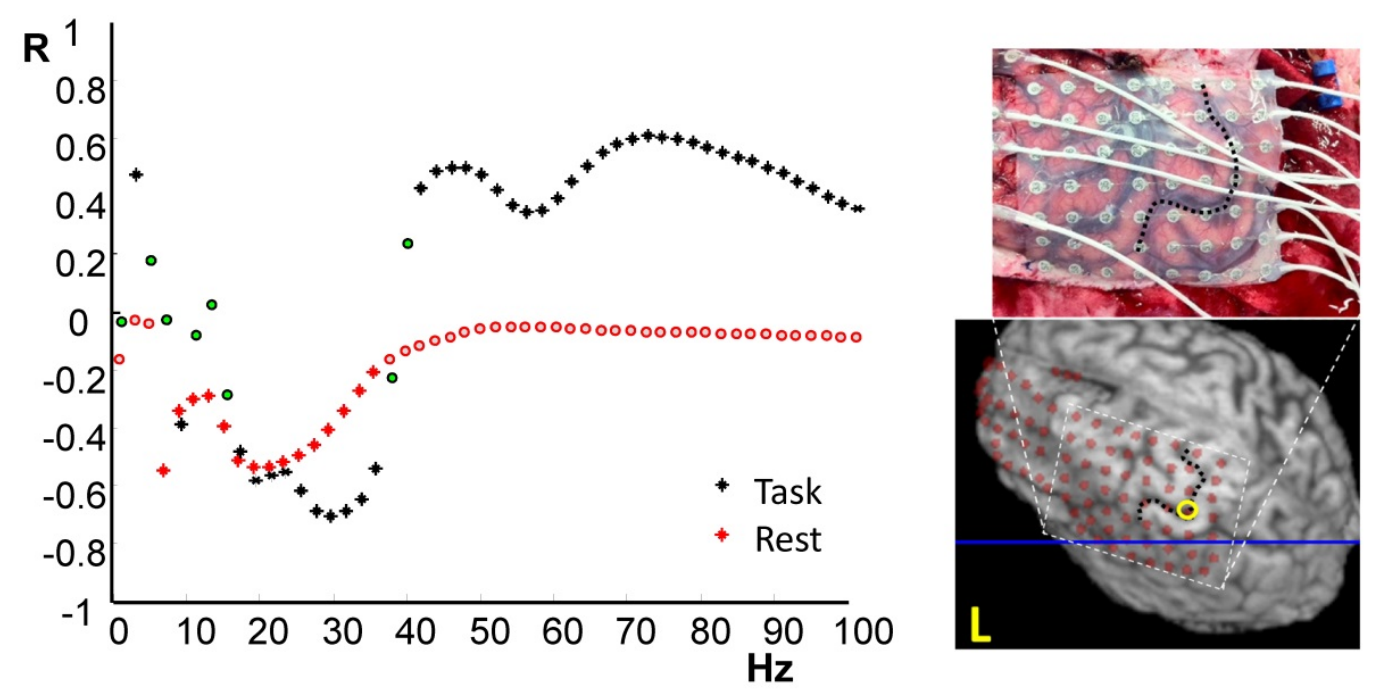

c.

Figure 2. Frequency-specific sensorimotor cortex correlations between ECoG and fMRI during task and rest

For each subject 1-3 (figures a-c) the fMRI signal from the fMRI task defined hand motor area was correlated with the co-localised ECoG data from a single electrode contact. The location of the electrode contact used is highlighted by a yellow circle on a reconstruction of the individuals cortical surface and ECoG contact locations a photo is also provided where available (subjects \#1 and \#3). Stars indicate significant correlation $p<0.001$ which corresponds to $p<0.05$ corrected for multiple comparisons, circles non-significant correlation values. Black represents the correlation during the task and red points correlation during rest. 


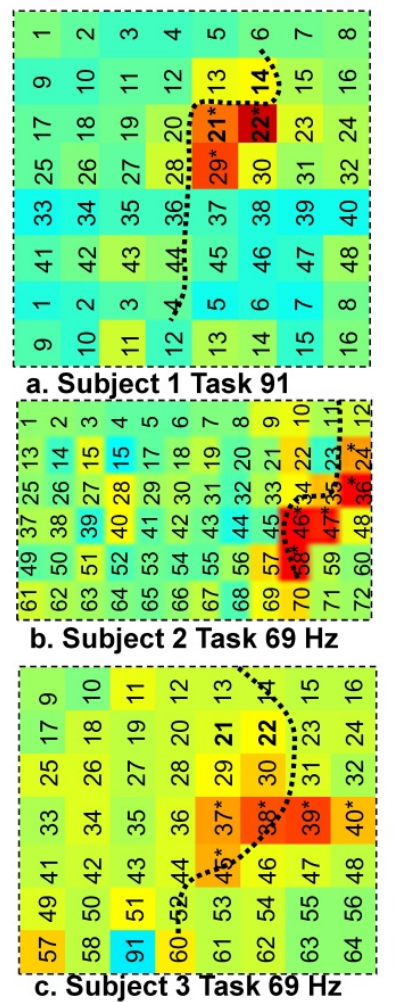

Figure 3. Spatial patte
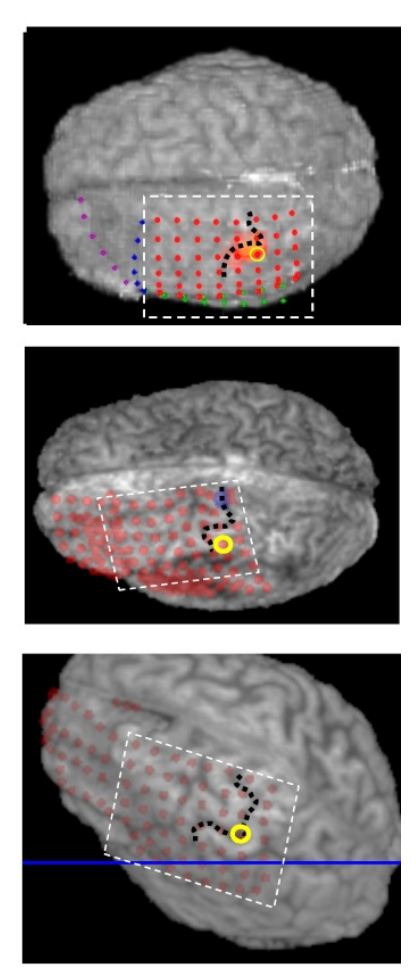

\section{tapping}

In a-c the correlation for each subject was mapped spatially over the cortex for the significant positive peak in correlation (gamma frequency range, see figures $2 a-c$ ) and negative peak in correlation (beta frequency range, see figures $2 d-f$ ).

The dotted line represents the position of the central sulcus and the numbers correspond to the electrodes numbering system (see table 2); * indicates significant correlation $p<0.001$ uncorrected.

Second, we investigated the coupling's spatial dimension, by mapping the fMRIECoG correlation across the ECoG grid. During the task (fig. 3) a region of very strong positive correlation at high gamma band frequencies was revealed at three post-central contacts located within $5 \mathrm{~mm}$ of the task-activated region (fig. 3a-c). In the beta range a strong negative correlation was seen over a much wider area of cortex including cortex that was more distant from the task-activated region in the pre- and post-central cortex (fig. 3d-f). During rest, the main feature was a significant (but weaker than during the task) negative correlation at frequencies in the alphabeta range in all three subjects (fig. 4). During the motor task in the beta band the region of negative correlation extended over a wide cortical area, extending beyond the primary motor cortex. 


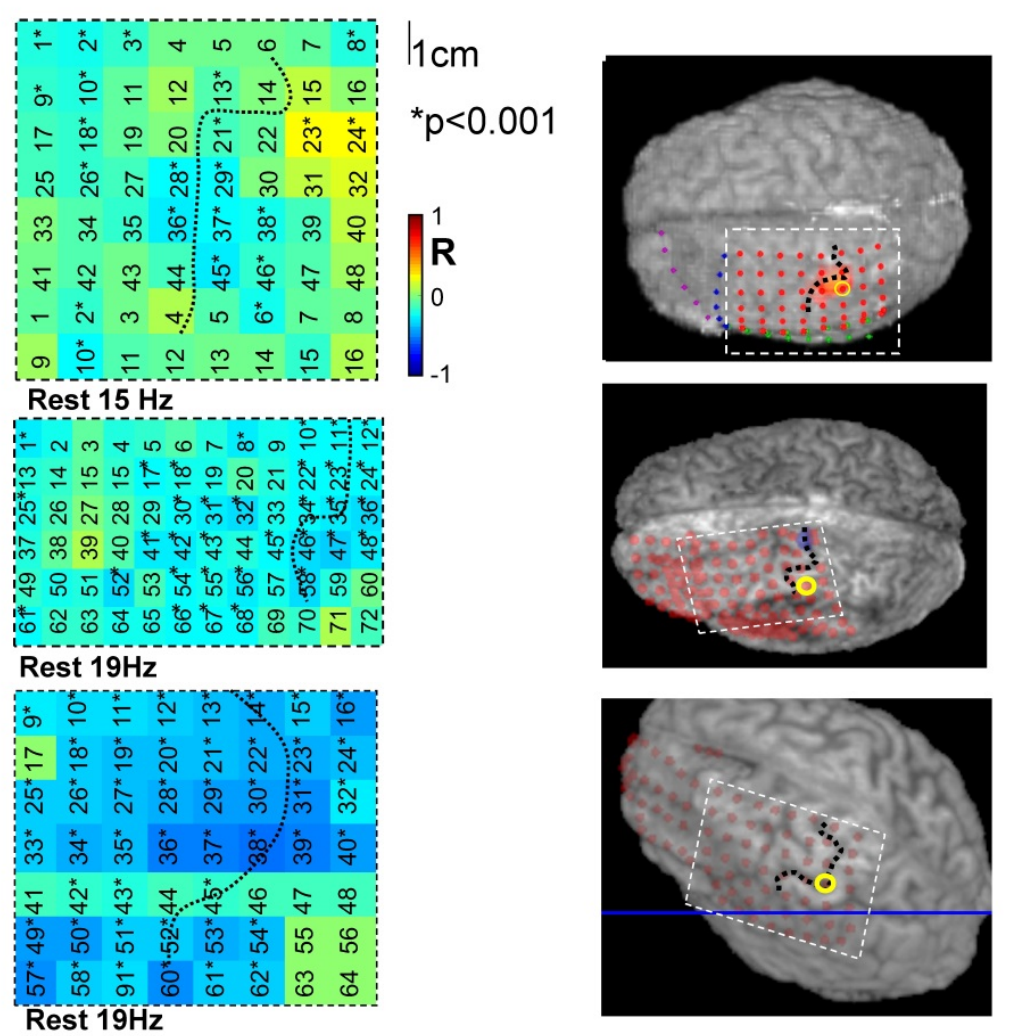

Figure 4. Spatial pattern of correlations between ECoG and fMRI during rest

In a-c the correlation for each subject was mapped spatially over the cortex for the significant negative peak in correlation (beta frequency range). Note positive correlations in the gamma range were not in general significant at rest and so were not spatially mapped.

\section{Comparison of frequency specific and cross-spectral EEG models of fMRI}

We aimed to determine the best EEG-based model of fMRI fluctuations, at rest and during the task. We compared a family of models for comparison, grouped as follows: firstly, single- versus multiple-predictor models to determine if the fMRI could be best modelled by a single EEG feature or was best modelled by a more complex representation. Secondly we compared models based on classical EEG frequency bands and compared them to cross-spectral EEG measures.

The individual classical frequency band-based fMRI predictors were beta, and high gamma power based on previous observations (7). Two multiple-predictor models were designed to summarise the entire spectrogram: first, a previously used model comprising of the power in each of the classical frequency bands (delta, theta, alpha, beta, low gamma and high gamma) (6); second, cross-spectral variation obtained by principal component analysis of spectrogram (the components explaining $90 \%$ of the EEG variance were used in the model, resulting in 10-18 regressors). All of these 
EEG predictors were convolved by the haemodynamic response function. Five crossspectral summary metrics were designed to embody alternative aspects of the EEG spectrogram as a single predictor of BOLD chnages. These consist of three spectral first order moments ('means'): the root mean squared frequency (so-called 'Kilner heuristic') (' $q_{R M S F}$ ') (13), a modified version of the latter that amplifies the influence of the dominant frequency (' $q_{M S F}$ '), the spectrogram centre of mass $\left(I_{C o f M}\right)$; and two spectral second order moments: about its mean, and about $40 \mathrm{~Hz}(140 \mathrm{~Hz})$ based on the point of inflection for the correlation between $f M R I$ signal and EEG in figure 2.). See the Appendix for definitions and the Supplementary Material section for simulations illustrating the cross-spectral single predictor characteristics.

\begin{tabular}{|c|c|c|c|c|c|c|}
\hline \multicolumn{7}{|c|}{ Motor task } \\
\hline \multirow[b]{2}{*}{ Model } & \multirow[b]{2}{*}{ \# Predictors } & \multirow{2}{*}{$\begin{array}{c}\text { Frequency } \\
\text { Range }\end{array}$} & \multirow[b]{2}{*}{$\# 1$} & \multirow[b]{2}{*}{$\# 2$} & \multirow[b]{2}{*}{ \#3 } & \multirow[b]{2}{*}{ sum } \\
\hline & & & & & & \\
\hline qmsf' & 1 & $0-100$ & 48.3 & 15.4 & 1.5 & 65.2 \\
\hline qrmsf & 1 & $0-100$ & 15.2 & -0.4 & 3.3 & 18.1 \\
\hline CofM & 1 & $0-100$ & -1.5 & -7.2 & 12.5 & 3.8 \\
\hline ICofM & 1 & $0-100$ & 1.0 & -3.6 & -10.6 & -13.2 \\
\hline $140 \mathrm{~Hz}$ & 1 & $0-100$ & 1.6 & -20.2 & -11.1 & -29.6 \\
\hline PCA & $10-18$ & $0-100$ & 35.1 & 23.2 & 18.3 & 76.5 \\
\hline beta & 1 & $13-31$ & 10.8 & 16.1 & 12.7 & 39.6 \\
\hline gammah & 1 & $53-99$ & 39.0 & 26.9 & 12.7 & 78.6 \\
\hline 6-band & 6 & $0-100$ & -46.4 & -49.0 & -57.8 & -153.2 \\
\hline \multicolumn{7}{|c|}{ Rest } \\
\hline qmsf' & 1 & $0-100$ & -5.8 & -5.8 & 40.7 & 29.1 \\
\hline qrmsf & 1 & $0-100$ & -5.7 & 0.6 & 19.8 & 14.7 \\
\hline CofM & 1 & $0-100$ & -5.1 & -6.6 & -4.7 & -16.4 \\
\hline ICofM & 1 & $0-100$ & -7.8 & -14.9 & -6.5 & -29.2 \\
\hline $140 \mathrm{~Hz}$ & 1 & $0-100$ & -7.7 & -19.1 & 19.1 & -7.7 \\
\hline PCA & $10-18$ & $0-100$ & 1.2 & 7.9 & 81.6 & 90.6 \\
\hline beta & 1 & $13-31$ & -5.9 & 6.2 & 55.3 & 55.6 \\
\hline gammah & 1 & $53-99$ & -7.3 & 4.0 & -15.0 & -18.3 \\
\hline 6-band & 6 & $0-100$ & -180.0 & -73.6 & -101.6 & -355.1 \\
\hline & \multicolumn{3}{|c|}{ multiple predictor model } & & & \\
\hline & cross spectro & al model & & & & \\
\hline
\end{tabular}

Table 1 Model evaluation

Model evidence is shown for each model and subject relative to a 'null model' containing only nuisance regressors (6 realignment parameters and a high pass cosine filter). A value of 3 is considered strong evidence (equivalent to $p<0.05$ ), we considered the results to be significant across individuals when the sum was $>9$ and each subject individually had a positive model evidence. These values are shown in bold. 
These comparisons were performed for the ECoG channel showing the highest correlation in the previous analysis (which in all subjects was within $5 \mathrm{~mm}$ of the task fMRI activation) and across all ECoG grid channels (figure 5) using empirical Bayesian model comparison (24).

\section{Motor task}

First, we wanted to determine the best EEG-derived predictor of BOLD in the primary sensorimotor hand area during the motor (finger-tap) task, for the ECoG channel near $(<5 \mathrm{~mm}$ Euclidian distance) to the $\mathrm{fMRI}$ activation in the primary sensorimotor cortex. We compared the log of the ratio of model evidences of each model to a model containing only nuisance effects (24) High gamma power was the best singlepredictor electrophysiological measure that best predicted $\mathrm{FMRI}$ changes during the motor task. The PCA model performed similarly well, despite its increased complexity (which is strongly penalised by the model comparison method used) and was therefore both the best cross-spectral and multiple-predictor model.

We note that the multiple-predictor model based on classical frequency bands performed poorly compared to the other models, in particular compared to the PCA model. This suggests that averaging within classical frequency bands destroyed significant predictive information.

Second, we examined the spatial distribution of model performance. The spatial maps of the log evidence for the best performing models in each category $q_{M S F}$, high beta- or gamma-band and PCA are shown in figure 5. The high gamma band and $q_{M S F}$, models are good predictors only for contacts that are highly co-localised, whereas the PCA model performs well from nearly all electrode contacts including in distal (hierarchically higher) brain areas. The beta band predictor was also a good model of BOLD changes over a wide region of cortex. This is consistent with the wider region of cortical inhibition during ipsilateral finger tapping. Taken together, our results strongly suggest that there is an electrographic representation of the task over a much wider region of cortex than that which shows strong fMRI changes - and that this representation is distributed in a spatially-structured way over frequencies. 


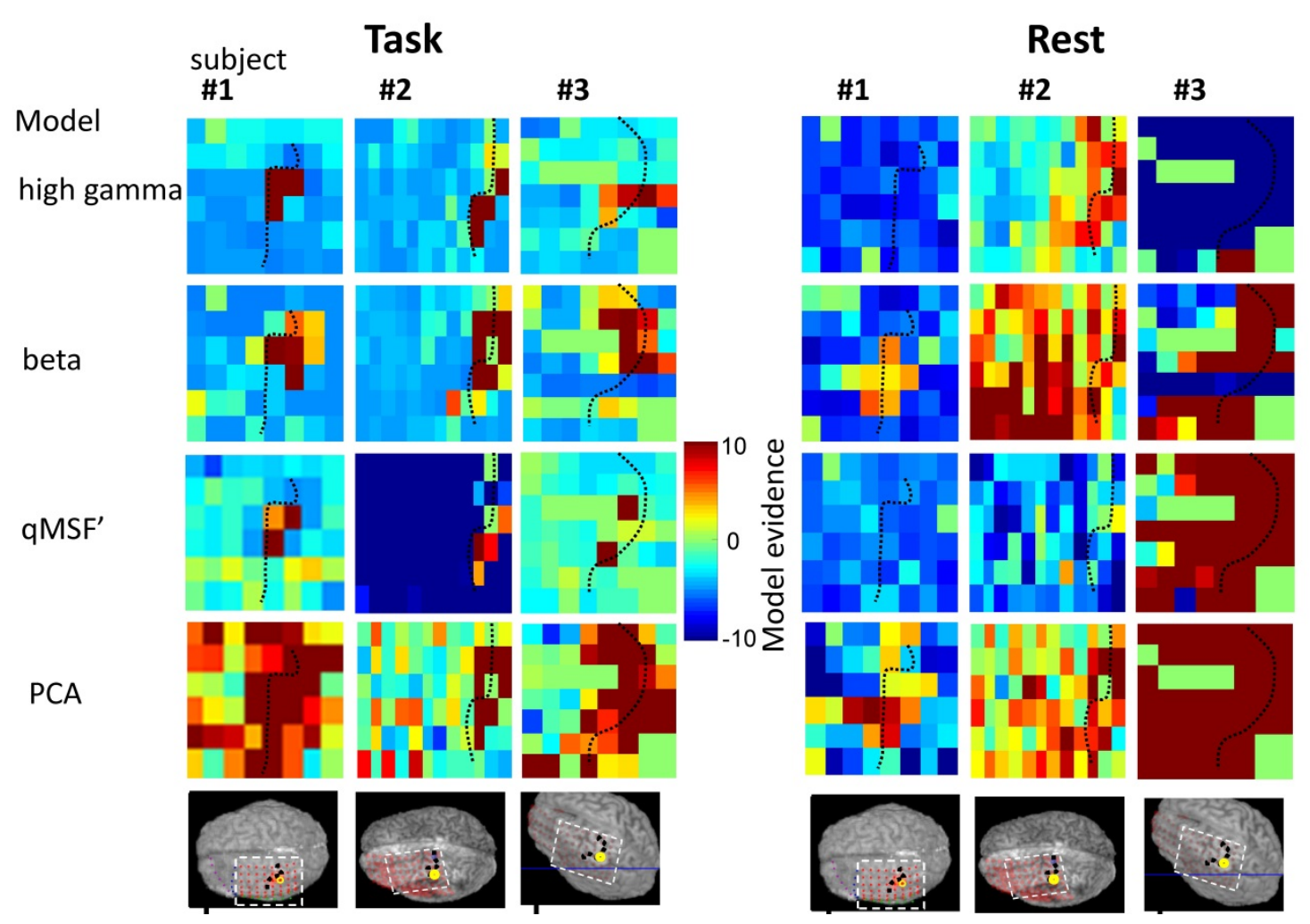

Figure 5. Spatial performance of different BOLD predictor models

In each plot the model evidence for a particular model and subject is plotted across the cortical grid. This was performed for the task (left hand side $4 \times 3$ panels) and rest (right hand side $4 \times 3$ panel). Each row represents a different model, dark red means strong evidence for the model and blue colours that the model is less predictive than the null (noise only) model.

\section{Resting state}

At rest, the pattern was more variable between subjects and model evidence lower across the board (Table 1). The PCA model performed best, and was the only metric to be better than the null model in all subjects. The best cross-spectral model single predictor model was the $\mathrm{q}_{\mathrm{MSF}}$, and the best individual frequency band model was beta band power. In patient \#1 although the PCA model was the best, model evidence was low. This may be due to the 'irritative zone' (generating interictal epileptic activity between seizures) overlapping with the primary sensorimotor region and disrupting normal ongoing resting-state activity. In the remaining subjects, there was strong evidence for the beta-band and PCA models explaining the BOLD signal fluctuations from the $f M R I$ defined sensorimotor cortex. The spatial distribution of model performance at rest (see figure 5) showed that a PCA model performed well over the primary sensorimotor cortex in all subjects, as did beta frequency band power. In 
contrast to the task data, high-gamma power was not a good BOLD predictor most likely due to its relative absence in this state.

\section{DISCUSSION}

We investigated the coupling between sensorimotor BOLD and electrophysiological signal variations in humans by mapping the correlation between the average fMRI signal from the hand sensorimotor region and frequency-specific ECoG signals. To do this, we used unique recordings of simultaneous electrophysiological signals on the surface of the human brain (ECoG) and whole-brain fMRI. In particular, the capability to record high-gamma activity over the motor cortex and fMRI together in humans - enabled us to study the coupling between electrophysiological and hemodynamic fMRI signals as a function of brain state and localization. The intracranial EEG signal has been shown to be of high fidelity and able to record high frequency activity (25).

\section{Local coupling}

In summary, we found task-related BOLD to reflect mainly high gamma-frequency power fluctuations in the local vicinity of $\mathrm{fMRI}$ activated region. In contrast, at rest, beta power in spatially distributed contacts best predicted (inversely) BOLD signal changes. The best BOLD predictor based on a single cross-spectral metric was ' $\mathrm{MSF}_{\text {' }}$ which models $\mathrm{fMRI}$ changes as shifts in the mean of the square of the spectral power such as those that can result from power fluctuations at specific frequencies (see the Appendix for details of the cross-spectral metrics behaviour). This is consistent with the notion of at least partially independent neuronal sources of EEG changes at specific frequencies (26). There were distinct peaks in the alpha-beta range with higher correlation (Fig 2), suggesting that there was a relationship between specific oscillatory activity and fMRI signal changes. In contrast at high gamma frequencies there was a high correlation across a broad frequency range. We found the PCAderived model to perform best across task and rest, and when considering the electrophysiological activity recorded over the entire ECoG grid. This model retains variance either from power changes at a given frequency or frequency shifts of any nature - it is simply a parsimonious description of the entire spectrogram. Taken together this may explain the PCA models large performance advantage over a model containing classical frequency band which have previously been suggested to be optimal (6).

\section{Spatial coupling aspects}


We found task-related BOLD to reflect mainly high gamma-frequency broad band power fluctuations in the vicinity of the BOLD response while beta band activity over a much larger cortical area was inversely correlated with the fMRI activity.

At rest, beta power in spatially distributed contacts (inversely) predicted BOLD signal changes but there was not the same focal positively collocated gamma activity. The beta-band activity was prevalent during rest and during contralateral hand movements, inversely correlating with $\mathrm{fMRI}$ over a wide area of sensorimotor cortex. This confirms it as general feature when attending to, or suppressing movement (27). In contrast gamma activity was highly localised in motor regions and only present during motor activity. It is possible that the relatively low power of the gamma activity made it more difficult to measure in the context of greater noise levels in ECoG recordings during $\mathrm{fMRI}$ although we have previously performed evaluation of this data(28).

\section{Novelty of our electrophysiological BOLD predictors}

Some of our findings confirm previous reports: for example, gamma-band activity was strongly coupled to BOLD $(4,5,29)$, but only during the task. Strong positive correlations in the gamma-band were found in conjunction with an inverse relationship at lower to mid frequency bands consistent with the idea that $f M R I$ signal increases reflect shifts in EEG power from low to high frequencies (13). These findings (fig. 2a-c) are consistent with studies in the auditory cortex using nonsimultaneous fMRI (pre-operative) and ECoG in two patients with epilepsy (5).

In the resting state, the coupling was reduced, although it had a similar form to the frequency dependence seen during the task (fig 2). Beta-band power was the most predictive frequency specific predictor of BOLD changes during rest (and was inversely correlated) potentially indexing the relative level of cortical inhibition, consistent with a scalp EEG-fMRI study (7).

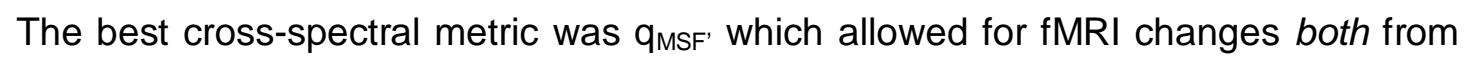
shifts in power from low to high frequencies and increased power within a frequency in the absence of other changes at specific frequencies (see supplementary material for details of the cross-spectral metrics). This might be expected given that different neuronal populations are thought to be responsible for generating these signals (26). We also evaluated multiple predictor models based on classical frequency bands and a cross-spectral model using PCA of the entire spectrogram. When considering all brain states and whether or not the ECoG channel was close to the $\mathrm{fMRI}$ region, we found the BOLD responses were best predicted by the PCA multi-spectral model. Because the PCA model is substantially more complex, it must explain far more 
BOLD variance than any other model to retain the greatest model evidence on marginal likelihood, given that our model comparison heavily penalises model complexity. Crucially, and in contrast to previous studies in the non-human primate visual cortex (6), our data does not support the multiple predictor model based on the classical frequency-bands as the best predictor of BOLD. This may be explained by the fact that our model comparison properly penalised model complexity - but could also be due to differences between brain regions and species.

Previous studies have found some variability in the neurophysiological features that best explain BOLD fMRI changes from both single spectral EEG frequencies, crossspectral models (8), or models containing several frequency bands (6). Crucially we have demonstrated that the results of these analyses are sensitive both to the subject's state and to the spatial relationship between the two modalities.

Our results suggest that focal fMRI changes are accompanied by widespread EEG changes. The EEG changes predicting $\mathrm{fMRI}$ were not limited to specific frequencies or a close co-localisation; therefore a cross-spectral PCA model is likely to be effective when looking at different brain regions that exhibit activity at different frequencies. From the reverse perspective this suggests that $f M R I$ changes can be linked to a plethora of EEG changes, and cannot be in general interpreted as relating to a single specific EEG feature. The exception to this rule was found during activity of primary cortex, where there was a close correspondence between high gamma power and fMRI, consistent with previous studies $(3-5,29)$. This is entirely consistent with predictive modelling of resting state connectivity in EEG and $\mathrm{fMRI}$, where EEG connectivity in all frequency bands could predict fMRI to a greater extent than fMRI could predict EEG based connectivity (30).

The ability of ECoG-fMRI to demonstrate the link between fMRI fluctuations and (oscillatory) neural activity should help refine biophysical models, particularly in accounting for emerging properties of neuronal populations (e.g. ref (31)) and for subsequent application in pathology (32). More generally, the characterisation of the coupling shown here, in different brain states, represents vital information for the interpretation of $\mathrm{fMRI}$ responses in terms of the underlying processing (1). For example, it can be crucial for informing and validating efforts to model the underlying distributed brain responses such as the effective connectivity between neuronal populations (1). There is increasing evidence of cortical layer specific spectral features of the EEG signal (26). This would provide a biophysical explanation of the potential independent contributions to BOLD changes (7). The strong predictive 
performance of the PCA model suggests that there are widespread cortical EEG changes that reflect focal BOLD signal changes and this complexity, if characterised could provide important new information, and avoid the misinterpretation of neurophysiological and $\mathrm{fMRI}$ comparisons.

\section{Epilepsy and ECoG-fMRI: feature for study and potential confound}

The patients studied suffer from epilepsy so that inferring behaviour in the healthy population requires some caution. However, electrode placement strategies are designed to test contrasting localisation hypotheses (devised following the results of non-invasive tests) and frequently include electrodes placed within or over regions presumed to be unaffected to map eloquent cortex. Intracranial EEG recordings in patients with epilepsy represent a unique opportunity to access a wide range of brain regions for study under conditions involving relatively normal physiology (e.g. ref (5)). The ECoG is exquisitely sensitive to local epileptic electrophysiological features, which should enable the effects of epilepsy to be accurately modelled and removed from studies aiming to make inferences about 'normal' brain function. This also provides an opportunity to map and understand $\mathrm{FMRI}$ changes associated with epileptiform events, including high frequency oscillations $(33,34)$ with unprecedented sensitivity, because we have demonstrated the ability to utilise high frequency ECoG data obtained with simultaneous fMRI. 


\section{Methods}

Three patients with refractory focal epilepsy were studied following a comprehensive safety assessment, approval from our local ethics committee (Joint UCL/UCLH), and written informed consent from the participants. Each patient underwent a tailored ECoG implantation (Table 2) based on the hypothesis generated from -long-term scalp video-EEG recording and other clinical data.

A carefully devised experimental protocol was followed to ensure patient safety, based on testing of the exact experimental configuration used for scanning and a large margin of safety to account for possible differences between in-vitro experiments and in-vivo studies (35-37). Following completion of the clinical ECoG recordings, cables connecting the electrodes to the amplifiers were replaced by customized $90 \mathrm{~cm}$ cables for simultaneous ECoG-fMRI recording, bundled, rerouted to the vertex of the head, re-bandaged and laid out precisely along the scanners central z-axis (37). All implanted electrodes were recorded from when amplifier capacity allowed, otherwise, targeted electrodes were selected (Table 2).

\section{Data acquisition}

We performed MRI with a 1.5T Siemens TIM Avanto scanner (Siemens, Erlangen, Germany) with a head transmit-receive coil, low SAR scans $(\leq 0.4 \mathrm{~W} / \mathrm{Kg}$ head average), exact electrode cable placement along the scanner and RF coils' Z-axis running towards the head end of the(37). The following scans were performed 1) localiser, 2) FLASH T1-volume, TR / TE / flip angle = $150 \mathrm{~ms} / 4.49 \mathrm{~ms} / 25^{\circ}$, 3) 10 minute gradient echo EPI (TR $3 \mathrm{~s} /$ TE $40 \mathrm{~ms} /$ flip angle $90^{\circ}, 38 \times 2.5 \mathrm{~mm}$ slices, 0.5 $\mathrm{mm}$ gap, $3 \times 3 \mathrm{~mm}$ in-plane resolution) in the resting state where the patients were instructed to lie still with their eyes closed 4) 10 minute gradient echo EPI where a visually cued opposing finger-to-thumb task was performed for $300 \mathrm{~s}$ with the same EPI parameters as above with $30 \mathrm{~s}$ blocks of left vs. right hand. In all subjects ECoG signals were recorded using an MR-compatible amplifier system (128 channels) (Brain Products, Munich, Germany) during fMRI acquisitions. The EEG recording system sampling at $5000 \mathrm{~Hz}$ was synchronized to the scanner's $20 \mathrm{kHz}$ gradient clock to allow direct correlation over time. The system was located at the head end of the scanner and ECG was recorded using a 16 bipolar channel ExG MR compatible system located at the patient's feet. Carbon fibre leads were used for the ECG recording in addition to spatial separation to prevent potential interactions between the intracranial electrodes and leads with the ECG; both were recorded using Brain Recorder (Brain Products, Gilching, Germany). Recordings sampled at $5000 \mathrm{~Hz}$ with subsequent filtering and down sampling to $250 \mathrm{~Hz}$. 
Table 2: Subject and ECoG recording information.

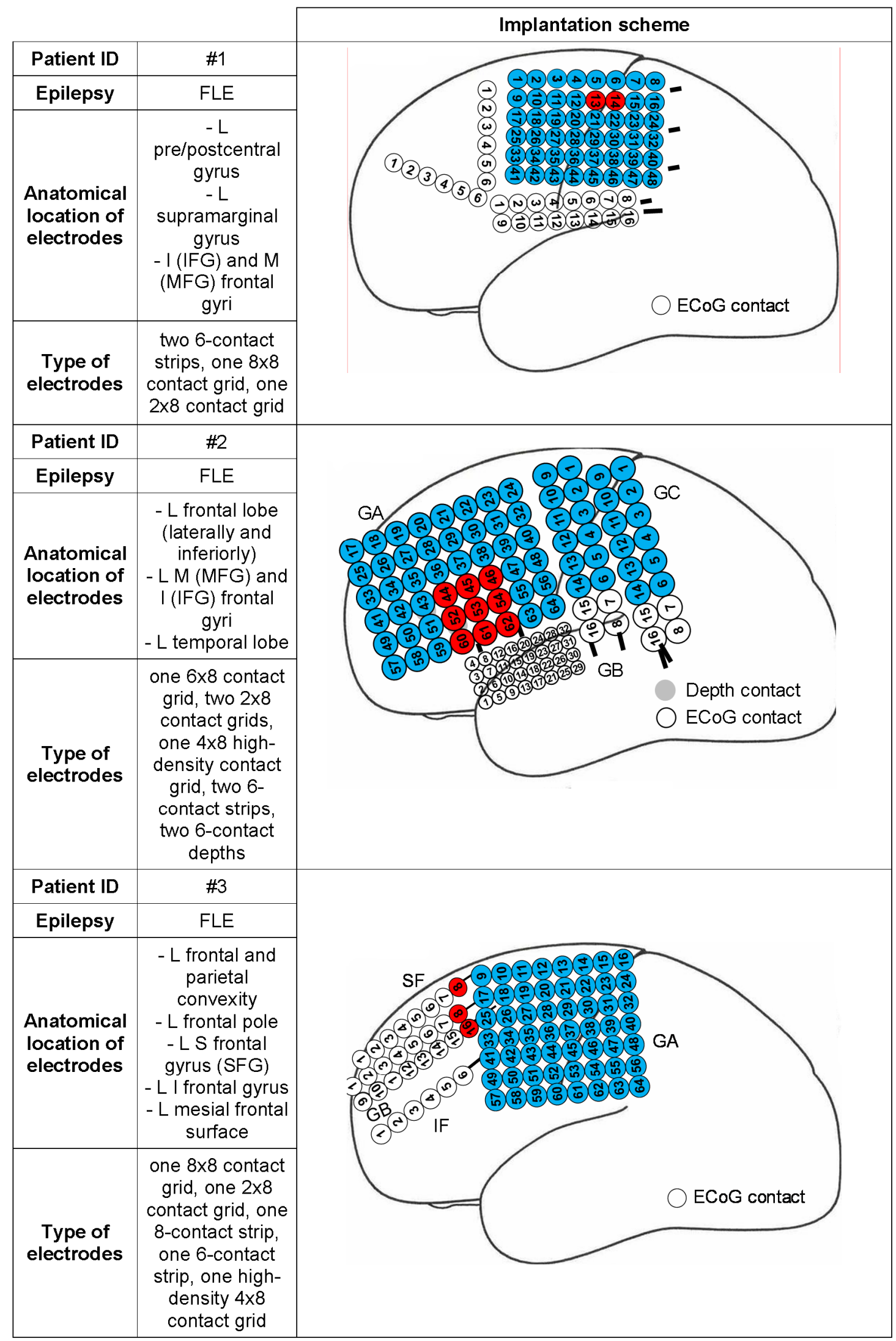

In red are the electrodes recording the seizure onset zone (we did not record those from patient \#3 during simultaneous ECoG-fMRI); in light blue are electrodes from 
which we recorded ECoG-fMRI in white (in addition to red electrode in patient \#3) are electrodes from which we did not record ECoG-fMRI.

\section{Data processing}

Scanning-related artefacts on EEG were removed using the Brain Analyser V1.3 (Brain Products, Munich, Germany) implementation of the template subtraction and filtering algorithm (38) and data was referenced to the average.

All EPI images were realigned to the first image and spatially smoothed (FWHM $8 \mathrm{~mm})$. The presence of significant $\mathrm{fMRI}$ changes correlated with the effect of interest (see below) was assessed voxel by voxel over the whole field of view using SPM software (www.fil.ion.ucl.ac.uk/spm) in Matlab (www.mathworks.com).

For the five-minute finger tap task, a standard block design was used in a general linear model - with motion modelled as the six realignment parameters and a voxelwise statistical threshold of $p<0.05$ family wise error corrected for multiple comparisons across voxels was applied to the ensuing SPM of t statistics.

The position of each electrode contact was determined on a CT scan (obtained for clinical purposes) co-registered to the space of the EPI data. The distance from each electrode contact to a cluster of fMRI activation was calculated as the minimum Cartesian distance over all voxels in the cluster.

Spectral analyses were performed for each electrode contact on the gradient artefact corrected ECoG data using a Morlet wavelet transform (39) as implemented in SPM8 (www.fil.ion.ucl.ac.uk/spm) with a wavelet factor value of 7 , to obtain time-frequency data for each electrode at frequencies 1,3,5.. $99 \mathrm{~Hz}$, giving time-frequency spectrograms at $2 \mathrm{~Hz}$ spectral sampling.

The primary hand sensorimotor region was defined as the fMRI task activated region. The realigned (but not spatially smoothed) data from this region was extracted and averaged before having first order drifts in $\mathrm{fMRI}$ signal removed. The time-frequency signals described above at frequencies $1,3,5 \ldots 99 \mathrm{~Hz}$ were convolved with the standard 'canonical' HRF in SPM before being re-sampled at the time corresponding to the start of each scan. Local correlations were calculated between each ECoGfrequency model and the measured $\mathrm{fMRI}$ response from the hand sensorimotor region. This was performed for data obtained both during the task and periods of rest (as described above). A significance threshold of $p<0.001$ was used, corresponding to a level of $p<0.05$ Bonferroni corrected for multiple comparisons across frequencies. This represents a very conservative threshold because the EEG has significant correlations over frequencies (whereas Bonferroni correction assumes independence between each frequency). Given that the data is also spatially smooth 
(spatially non-independent) (40) and the initial threshold is very conservative we did not correct further for multiple comparisons across electrode contacts.

For the model comparison for each channel EEG, the time-frequency data described above was 1) averaged based on frequency-bands (delta: 1, $3 \mathrm{~Hz}$; theta: $5,7 \mathrm{~Hz}$; alpha: 9, $11 \mathrm{~Hz}$; beta: $13,15, \ldots 31$; gamma Low: $33,35, \ldots 51 \mathrm{~Hz}$; gamma high: 53 , $55 \ldots 99 \mathrm{~Hz}$ ) before being convolved with the canonical hemodynamic response function (HRF) and re-sampled at the start of each scan volume (3s temporal resolution).

Models were built with individual spectral band regressors (single predictors) and the power in all of the six chosen frequency-bands (multiple predictor model). Motion was modelled as the 6 realignment parameters and slow temporal drifts were modelled using a cosine filter or order 1.

We calculated each of the family of cross-spectral single predictor models described in the Appendix and compared them to the models based on classical frequency bands described above. Finally, we used created a multiple predictor cross-spectral model using a parsimonious data driven model of the complete spectrogram by performing a Principal Component Analysis (PCA) and taking the components that described $>90 \%$ of the variance. Typically this yielded the most complex model with 10-18 repressors giving an indication of the rich spectral information content of the intracranial EEG data.

\section{Model comparison}

Bayesian model comparison (as implemented in spm_PEB.m, spm8 (24)) was used to compare the different EEG-derived models ability to predict the BOLD signal changes. The model evidence (log evidence) for each of the EEG-derived models described above was calculated and the relative evidence compared to the null model (containing motion and slow temporal drifts). In this context a value of 3 is equivalent to a $5 / 100$, and 20 to $1 / 1000$ of the model being better by chance (i.e. equivalent to a $p$-value of 0.05 and 0.001 respectively in classical statistics). It should be noted that the models are penalised for complexity and therefore the evidence scores the model that explains the most variance given the complexity; therefore allowing for a conservative comparison of models with increased complexity. 


\section{ACKNOWLEDGEMENTS}

We would like to thank Catherine Scott, Dr. Beate Diehl and Philip Allen in the Epilepsy Telemetry Unit, Prof. John Duncan in the Epilepsy Department and Lisa Strycharczuk, Alison Duncan, Catherine Green, Prashanth Kesara, Bruce Metheringham and Dr. Laura Mancini in the Lysholm Department of Neuroradiology at the National Hospital for Neurology and Neurosurgery, University College London Hospitals NHS Foundation Trust, London for their help, guidance and facilitation of this work. Dr. Rachel Thornton from the Department of Clinical and Experimental Epilepsy, UCL Institute of Neurology, London. Will Penny from the WTCN, UCL Institute of Neurology, London for advice. Robert Störmer from Brain Products, $\mathrm{GmbH}$, Gilching, Germany for technical advice about EEG hardware and EEG artifact correction. S Vulliemoz is supported by the Swiss National Science Foundation grant SNF 141165 and 140332. We acknowledge the financial support of the UK Medical Research Council (MRC grant G0301067). This work was undertaken at UCLH/UCL who received a proportion of funding from the Department of Health's NIHR Biomedical Research Centres funding scheme.

\section{AUTHOR CONTRIBUTIONS}

DWC was primarily responsible for designing and performing the experiments, for data analysis, and for writing the manuscript. SV, UC assisted in conducting the invivo experiments, the data analysis (particularly for neurophysiological assessment, EEG quality and correction), and in writing the manuscript. RR, TM and SP assisted in data analysis and figure generation (particularly for image coregistration, electrode localization and visualisation) and reviewed the manuscript. LL conceived the project and had overall responsibility for its management (scientific, ethical and financial) and assisted in data interpretation and writing the manuscript. TM, MR, and KF provided assistance in data analysis and interpretation and assisted writing the manuscript. 


\section{References}

1. Friston, K. J. (2009) Modalities, modes, and models in functional neuroimaging Science 326, 399-403.

2. Logothetis, N. K. (2008) What we can do and what we cannot do with fMRI Nature 453, 869-878.

3. Logothetis, N. K., Pauls, J., Augath, M., Trinath, T. \& Oeltermann, A. (2001) Neurophysiological investigation of the basis of the fMRI signal Nature 412, 150-157.

4. Niessing, J. et al. (2005) Hemodynamic signals correlate tightly with synchronized gamma oscillations Science 309, 948-951.

5. Mukamel, R. et al. (2005) Coupling between neuronal firing, field potentials, and FMRI in human auditory cortex Science 309, 951-954.

6. Goense, J. B. \& Logothetis, N. K. (2008) Neurophysiology of the BOLD fMRI signal in awake monkeys Curr. Biol. 18, 631-640.

7. Scheeringa R, Fries P, Petersson KM, Oostenveld R, Grothe I, Norris DG, Hagoort P, Bastiaansen MC. (2011) Neuronal dynamics underlying high- and low-frequency EEG oscillations contribute independently to the human BOLD signal.Neuron. Feb 10;69(3):572-83.

8. Rosa, M. J., Kilner, J., Blankenburg, F., Josephs, O. \& Penny, W. (2010) Estimating the transfer function from neuronal activity to BOLD using simultaneous EEG-fMRI Neuroimage. 49, 1496-1509.

9. Conner CR, Ellmore TM, Pieters TA, DiSano MA, Tandon N. (2011): Variability of the relationship between electrophysiology and BOLD-fMRI across cortical regions in humans. J Neurosci 31(36):12855-65. (16) Biswal, B., Yetkin, F. Z., Haughton, V. M. \& Hyde, J. S. (1995)

10. Hermes D, Miller KJ, Vansteensel MJ, Aarnoutse EJ, Leijten FS, Ramsey NF. (2012): Neurophysiologic correlates of $\mathrm{fMRI}$ in human motor cortex. Hum Brain Mapp. 33(7):1689-99.

11. Murta T, Leite M, Carmichael DW, Figueiredo P, Lemieux L. Electrophysiological correlates of the BOLD signal for EEG-informed fMRI. Hum Brain Mapp. 2015 Jan;36(1):391-414.

12. Canolty RT1, Knight RT. (2010) The functional role of cross-frequency coupling. Trends Cogn Sci. 2010 Nov;14(11):506-15.

13. Kilner, J. M., Mattout, J., Henson, R. \& Friston, K. J. (2005) Hemodynamic correlates of EEG: a heuristic Neuroimage. 28, 280-286.

14. Rubino, D., Robbins, K. A. \& Hatsopoulos, N. G. (2006) Propagating waves mediate information transfer in the motor cortex Nat. Neurosci. 9, 1549-1557.

15. Akam T, Kullmann DM. Oscillations and filtering networks support flexible routing of information. Neuron. $2010 \mathrm{Jul}$ 29;67(2):308-20.

16. Miller KJ, Hermes D, Honey CJ, Hebb AO, Ramsey NF, Knight RT, Ojemann JG, Fetz EE. (2012). Human motor cortical activity is selectively phase-entrained on underlying rhythms. PLoS Comput Biol. 2012;8(9):e1002655.

17. Tao, J. X., Ray, A., Hawes-Ebersole, S. \& Ebersole, J. S. (2005) Intracranial EEG substrates of scalp EEG interictal spikes Epilepsia 46, 669-676.

18. Santiuste, M. et al. (2008) Simultaneous magnetoencephalography and intracranial EEG registration: technical and clinical aspects J. Clin. Neurophysiol. 25, 331-339.

19. Magri C, Schridde U, Murayama Y, Panzeri S, Logothetis NK. (2012): The amplitude and timing of the BOLD signal reflects the relationship between local field potential power at different frequencies. J Neurosci 32(4):1395-407.

20. Nir, Y. et al. (2008) Interhemispheric correlations of slow spontaneous neuronal fluctuations revealed in human sensory cortex Nat. Neurosci. 11, 1100-1108. 
21. Schölvinck ML, Maier A, Ye FQ, Duyn JH, Leopold DA (2010) Neural basis of global resting-state fMRI activity. Proc Natl Acad Sci U S A 107:10238-10243.

22. Friston, K. J. et al. (1995) Analysis of fMRI time-series revisited Neuroimage. 2, 4553.

23. Biswal, B., Yetkin, F. Z., Haughton, V. M. \& Hyde, J. S. (1995) Functional connectivity in the motor cortex of resting human brain using echo-planar MRI Magn Reson. Med. 34, 537-541.

24. Penny WD., Stephan KE., Mechelli A., and Friston KJ. (2004). Comparing dynamic causal models Neuroimage. 22 1157-1172.

25. Saignavongs $M$, Ciumas $C$, Petton $M$, Bouet $R$, Boulogne $S$, Rheims $S$, Carmichael DW, Lachaux JP, Ryvlin P. (2017) Neural Activity Elicited by a Cognitive Task can be Detected in Single-Trials with Simultaneous Intracerebral EEG-fMRI Recordings. Int J Neural Syst. Feb;27(1):1750001.

26. Buffalo, E. A., Fries, P., Landman, R., Buschman, T. J., \& Desimone, R. (2011). Laminar differences in gamma and alpha coherence in the ventral stream. Proceedings of the National Academy of Sciences, 108(27), 11262-11267.

27. Engel, A. K. \& Fries, P. (2010) Beta-band oscillations--signalling the status quo? Curr. Opin. Neurobiol. 20, 156-165.

28. Murta T, Chaudhary UJ, Tierney TM, Dias A, Leite M, Carmichael DW, Figueiredo P, Lemieux L.Phase-amplitude coupling and the BOLD signal: A simultaneous intracranial EEG (icEEG) - $f M R I$ study in humans performing a finger-tapping task. Neuroimage. 2017 Feb 1;146:438-451.

29. Lachaux JP, Fonlupt P, Kahane P, Minotti L, Hoffmann D, Bertrand O, Baciu M. Relationship between task-related gamma oscillations and BOLD signal: new insights from combined fMRI and intracranial EEG. Hum Brain Mapp. 2007 Dec;28(12):136875.

30. Deligianni F, Centeno M, Carmichael DW, Clayden JD. (2014). Relating resting-state $\mathrm{fMRI}$ and EEG whole-brain connectomes across frequency bands. Front Neurosci.

31. Kopell, N., Ermentrout, G. B., Whittington, M. A. \& Traub, R. D. (2000) Gamma rhythms and beta rhythms have different synchronization properties Proc. Natl. Acad. Sci. U. S. A 97, 1867-1872.

32. Uhlhaas, P. J. \& Singer, W. (2006) Neural synchrony in brain disorders: relevance for cognitive dysfunctions and pathophysiology Neuron 52, 155-168.

33. Bragin, A., Engel, J., Jr., Wilson, C. L., Fried, I. \& Buzsaki, G. (1999) High-frequency oscillations in human brain Hippocampus 9, 137-142.

34. Zijlmans, M., Jacobs, J., Zelmann, R., Dubeau, F. \& Gotman, J. (2009) High-frequency oscillations mirror disease activity in patients with epilepsy Neurology 72, 979-986.

35. Carmichael, D. W. et al. (2010) Feasibility of simultaneous intracranial EEG-fMRI in humans: a safety study Neuroimage. 49, 379-390.

36. Carmichael, D. W. et al. (2008) Safety of localizing epilepsy monitoring intracranial electroencephalograph electrodes using MRI: radiofrequency-induced heating J. Magn Reson. Imaging 28, 1233-1244.

37. Carmichael DW, Vulliemoz S, Rodionov R, Thornton JS, McEvoy AW, Lemieux L. (2012) Simultaneous intracranial EEG-fMRI in humans: protocol considerations and data quality. Neuroimage. 2012 Oct 15;63(1):301-9.

38. Allen, P. J., Josephs, O. \& Turner, R. (2000) A method for removing imaging artifact from continuous EEG recorded during functional MRI Neuroimage. 12, 230-239.

39. Tallon-Baudry, C., Bertrand, O., Peronnet, F. \& Pernier, J. (1998) Induced gammaband activity during the delay of a visual short-term memory task in humans $\mathrm{J}$. Neurosci. 18, 4244-4254. 
bioRxiv preprint doi: https://doi.org/10.1101/237198; this version posted December 21, 2017. The copyright holder for this preprint (which

was not certified by peer review) is the author/funder. All rights reserved. No reuse allowed without permission.

40. Kilner, J. M., Kiebel, S. J. \& Friston, K. J. (2005) Applications of random field theory to electrophysiology Neurosci. Lett. 374, 174-178. 


\section{Appendix}

\section{Cross-spectral single predictors of BOLD: introduction and theory}

In this section we set out to derive and test a family of metrics inspired by the previously proposed heuristic of Kilner et al (13); these can be categorised as first and second moment measures of the ECoG spectrum.

The Kilner heuristic attempts to capture the basic assumption that BOLD reflects the position of the 'root mean squared EEG frequency' (RMSF):

$$
\begin{gathered}
q_{R M S F}(t)=\sqrt{\sum_{f=1}^{n_{f}} f^{2} \tilde{P}(f, t)} \\
\tilde{P}(f, t)=P(f, t) / \frac{1}{f} \sum_{f=1}^{n_{f}} P(f, t)
\end{gathered}
$$

Where $f$ are the frequencies, $n_{f}$ the spectrum's upper bound and $\tilde{P}$ is the spectral power of the EEG time series normalised by the total spectral power (at each time point).

This metric was found to be a reasonable explanatory of the BOLD response in a combined scalp EEG-fMRI study (8) albeit over a lower frequency range (1-30Hz). Due to this normalisation by the total power at each time point an isolated change in power at a given frequency, if independent of other spectral changes, will either cause a positive or negative change in the $q_{R M S F}$ metric depending on its frequency relative to the spectral centre of mass. Observations regarding the importance of both synaptic and spiking activity (34), and our experience of intracranial EEG data led us to hypothesise that the behaviour of this metric might not account for these plausible spectral changes.

We therefore developed a family of related metrics that embody a range of possible BOLD-spectrum behaviours. Firstly, we modified the first moment equation $A 1$ to increase the influence of the dominant frequency as follows:

$$
q_{M S F^{\prime}}(t)=\sum_{f=1}^{n_{f}} f^{2} \hat{P}(f, t)
$$

where $\hat{P}$ is the power at each time point normalised by the mean power at each frequency (across all time windows) and the total power in each spectrum (at each time window). 


$$
\hat{P}(f, t)=\tilde{P}(f, t) / \frac{1}{t} \sum_{t=1}^{n_{t}} P(f, t)
$$

In addition, we calculated the first moment of the frequency (spectral mean) and two second moments, firstly about the spectral mean, and secondly about $40 \mathrm{~Hz}$ (based on our observations of BOLD correlations to individual frequencies fig. 2a-c).

$$
\begin{aligned}
& f_{\text {CofM }}=\sum_{f=1}^{n_{f}} P(f, t) \cdot f \\
& I_{\text {CofM }}=\sqrt{\sum_{f=1}^{n_{f}}\left(f-f_{\text {CofM }}\right)^{2} \cdot P(f, t)} \\
& I_{40 \mathrm{~Hz}}=\sqrt{\sum_{f=1}^{n_{f}}(f-40)^{2} \cdot P(f, t)}
\end{aligned}
$$

\section{Supplementary Material}

\section{Simulation of 'heuristic' measures: methods}

To better characterise and illustrate the behaviour of the metrics in the Appendix we performed simulations of different patterns of spectral fluctuations. Discrete spectra were created with 1024 points within a range of $1-129 \mathrm{~Hz}$. The power at each frequency was determined by the addition of a $1 / f$ distribution and two Gaussian peaks were added to this spectrum at a 'low' $10 \mathrm{~Hz}$ and 'high' $60 \mathrm{~Hz}$ frequency with full width at half maximum of $4 \mathrm{~Hz}$. In order to simulate dynamic processes, we first increased the amplitude of the power at these frequencies up to a $100 \%$ of the power at that frequency in the $1 / f$ spectrum (see figure S1). Secondly, each peak was shifted in frequency upwards in steps of $\sim 0.1 \mathrm{~Hz}$ from $0-8 \mathrm{~Hz}$. The expected BOLD response was then determined using the range of 'heuristic' measures derived in the Appendix.

\section{Results}

The simulated spectra containing changes in peak amplitude are illustrated in figure S1. The simulated spectra containing changes in peak frequency are illustrated in figure S2. The corresponding predicted BOLD changes generated by the different 'heuristic' measures are shown in figure S3. For changes in peak amplitude (and therefore power) at a given frequency (top row of fig. S3), the $\mathrm{q}_{\mathrm{RMSF}}$ model predicts BOLD increases associated with increased power at high frequencies, and decreases with increased low frequency power. This is in contrast the $\mathrm{q}_{\mathrm{MSF}}$, which 
predicts BOLD increases with either increased low or high frequency power, although the predicted BOLD increase is greater at higher frequencies. The CofM metric is similar to the $\mathrm{q}_{\mathrm{RMSF}}$ metric in its behaviour, with the distinction that the ICofM predicts an equal change in BOLD for the same percentage change at $10 \mathrm{~Hz}$ or $60 \mathrm{~Hz}$. The $\mathrm{I}_{40 \mathrm{~Hz}}$ metric predicts BOLD increases with increased power at 10 or $60 \mathrm{~Hz}$, with a greater effect at $10 \mathrm{~Hz}$ than $60 \mathrm{~Hz}$. For the heuristic metric $q_{\mathrm{RMSF}}(13)$ frequency shifts in peaks at low and high frequencies predict a BOLD increase, with a greater effect at lower frequencies. The $\mathrm{q}_{\mathrm{MSF}}$, metric similarly shows a BOLD increase for a shift to higher frequencies of either a low or high frequency peak with a greater effect at high frequency.

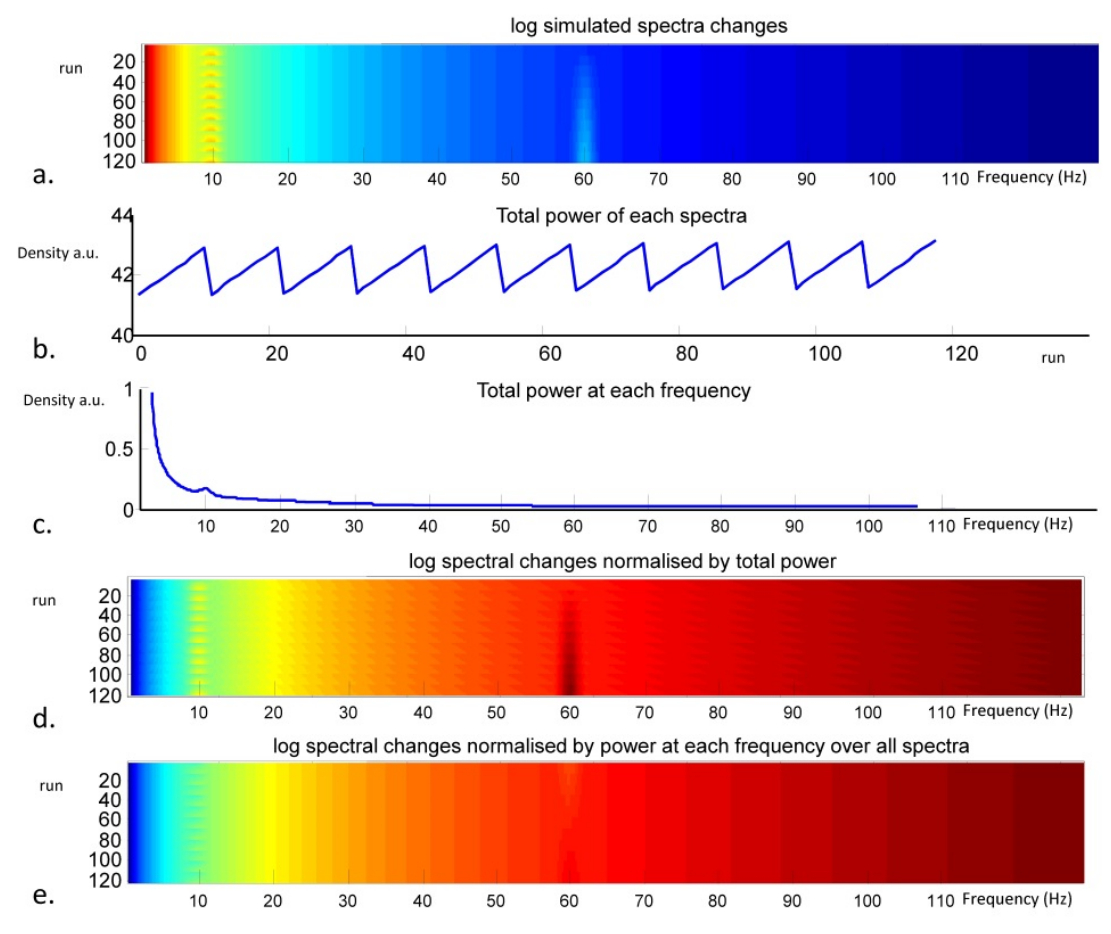

\section{Figure S1 Simulated spectra varying peak amplitude}

Spectra were simulated as a 1/f spectrum with two peaks at 10 and $60 \mathrm{~Hz}$. In each spectra the amplitude was altered (a). The variability in the total power for each simulated spectrum is shown (b). The average spectrum is calculated (c). The effect of normalising by the total power is shown in (d) and normalising by the average power at each frequency (e). 


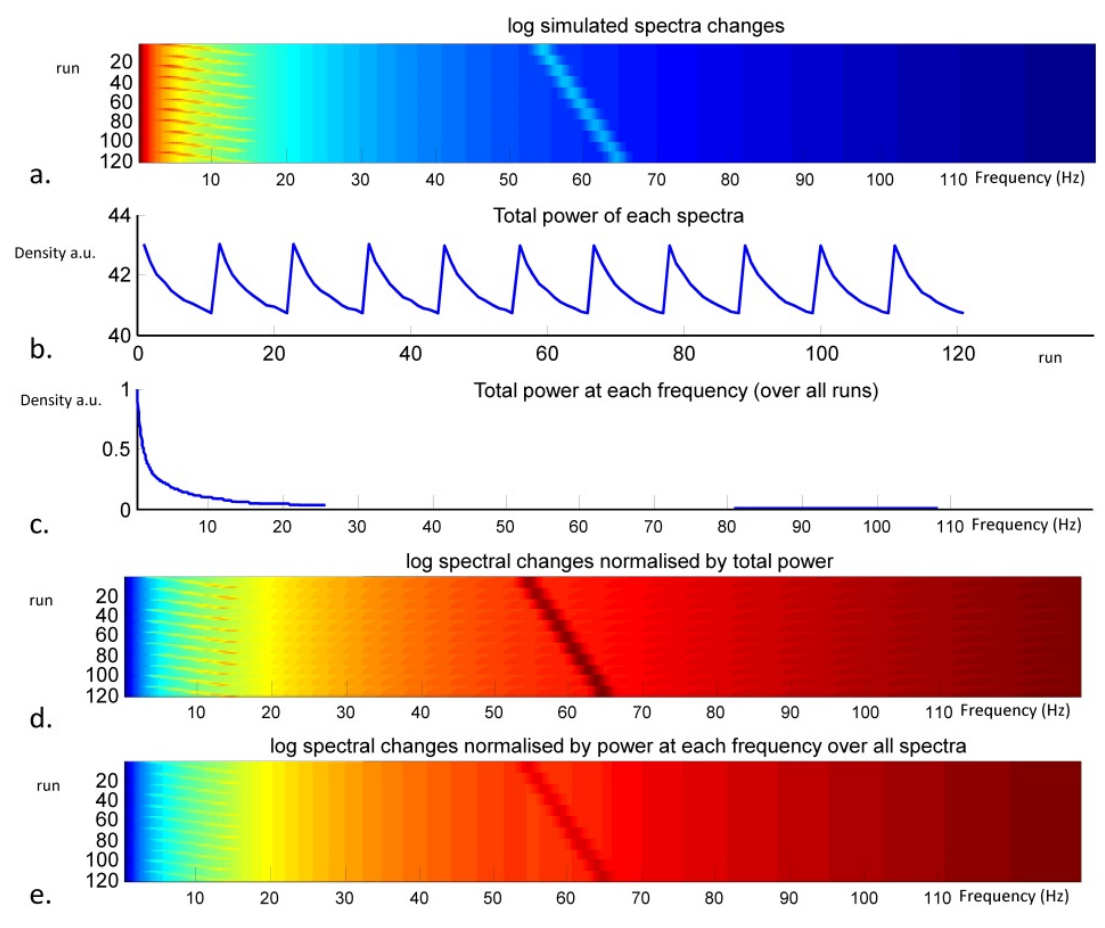

\section{Figure S2 Simulated spectra varying peak frequency}

Spectra were simulated as a 1/f spectrum with two peaks at 10 and $60 \mathrm{~Hz}$. In each spectra the peak frequency was altered (a). The variability in the total power for each simulated spectrum is shown (b). The average spectrum is calculated (c). The effect of normalising by the total power is shown in (d) and normalising by the average power at each frequency (e).

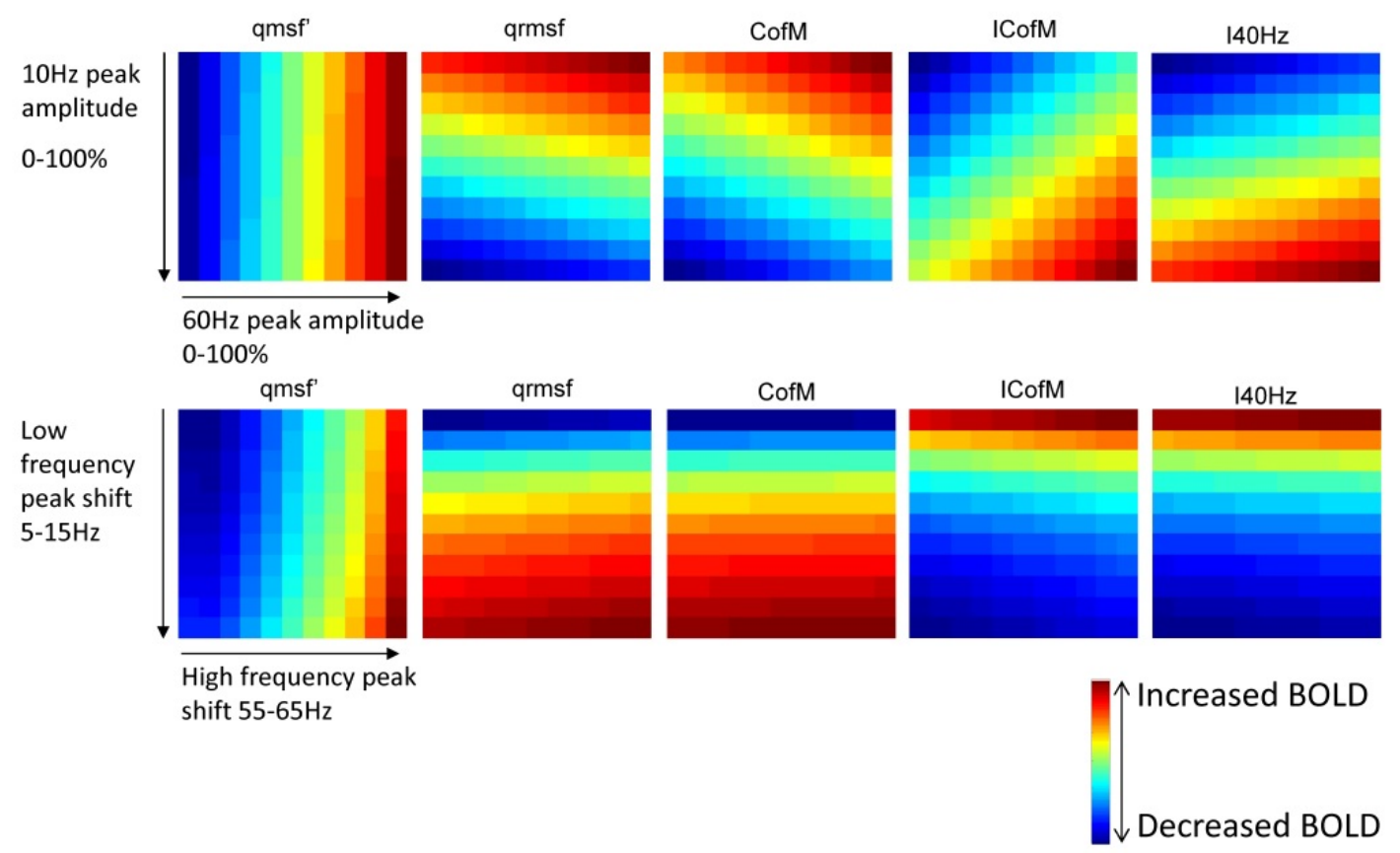

Figure S3 Simulated BOLD changes predict by different cross-spectral metrics 
The predicted responses by each of the cross spectral predictors defined in appendix 1 to the simulated spectral changes are shown. The top row shows the predicted BOLD response to changes in peak amplitude (spectra in fig. S1) for each metric and the bottom row the BOLD response predicted to changes in peak frequency (spectra in fig. S2). 